\title{
THE WORKS OF HUBERT HOWE BANCROFT
}

I. The Native Races of the Pacific States I-Wild Tribes

II. The Native Races of the Pacific States II-Civilized Nations

III. The Native Races of the Pacific States III-Mytbology

IV. The Native Races of the Pacific States IV-Antiquities

V. The Native Races of the Pacific States V-Primitive History

The History of the Pacific States

VI. Central America I. . . . . . . . . . . . . 1501-1530

VII. Central America II . . . . . . . . . . . . . . . . 1530-1800

VIII. Central America III. . . . . . . . . . . . . 1801-1887

IX. Mexico I . . . . . . . . . . . . . . . 1516-1521

X. Mexico II ...................... 1521-1600

XI. Mexico III . . . . . . . . . . . . . . . . 1600-1803

XII. Mexico IV ....................... 1804-1824

XIII. Mexico V .....................1824-1861

XIV. Mexico VI..................1861-1887

XV. Nortb Mexican States I. . . . . . . . . . . 1531-1800

XVI. Nortb Mexican States and Texas II . . . . . . . 1801-1889

XVII. Arizona and New Mexico............ 1530-1888

XVIII. California I . . . . . . . . . . . . . . . . 1542-1800 
The Works of Hubert Howe Bancroft

XIX. California II .....................1801-1824

XX. California III......................1825-1840

XXI. California IV .....................1840-1845

XXII. California V .....................1846-1848

XXIII. California VI ..................... 1848-1859

XXIV. California VII...................... 1860-1890

XXV. Nevada, Colorado and Wyoming ........1540-1888

XXVI. Utab ........................1540-1886

XXVII. Nortbwest Coast I.................. 1543-1800

XXVIII. Nortbwest Coast II................1800-1846

XXIX. Oregon I....................... 1834-1848

XXX. Oregon II .......................1848-1888

XXXI. Wasbington, Idabo and Montana ......... 1845-1889

XXXII. Britisb Columbia...................1792-1887

XXXIII. Alaska ........................1730-1885

XXXIV. California Pastoral ...................1769-1848

XXXV. California Inter Pocula ................1848-1856

XXXVI. Popular Tribunals I..................1851

XXXVII. Popular Tribunals II..................1856

XXXVIII. Essays and Miscellany

XXXIX. Literary Industries 
